\title{
Einleitende Worte der Herausgeberin der Reihe
}

Der nun 85. Band der traditionellen MPK-Reihe rückt neue Forschungen zu Boiern und Tauriskern in den Mittelpunkt dieser Latènestudien. Die insgesamt 14 hier zusammengestellten Beiträge stammen aus einer 2012 veranstalteten Tagung beim Oberleiserberg, einem traditionellen Zentrum für Latèneforschung in Ostösterreich. Die beiden Organisatoren und Herausgeber des Bandes, Maciej Karwowski und Peter Ramsl, haben aktuelle Forschungen zu vier Schwerpunkten zusammengestellt und namhafte Autoren/Autorinnen dafür gewinnen können. So ist den Herausgebern ein Band gelungen, der neueste und durchaus kritische Studien und Diskussionen zu verschiedenen Aspekten ostkeltischer Kultur beinhaltet. Die vier zentral behandelten Themen Taurisker, Boier, allgemeine Fragen zu den Ostkelten und Interaktion im mittleren Donauraum zeigen die ungebrochene Bedeutung materieller und typologischer Studien für die mitteleuropäische Latèneforschung, wobei Münzen, Fibeln, Knotenringe und Keramiktypen eine traditionell große Rolle in der weiteren soziokulturellen Interpretation spielen. Zusätzliche archäologische Quellen, wie beispielsweise Grubenhäuser oder neue Siedlungen, werden in weiteren Beiträgen behandelt. Die Intensität von Interaktion zwischen Regionen und ihr Einfluss auf lokale Entwicklungen werden in verschiedenen Beiträgen und unter unterschiedlichen Gesichtspunkten behandelt. Im generellen konzeptionellen Verständnis von der Einbettung materieller Kultur in historisch überlieferte Rahmendaten werden dabei Kontakte und Verbindungen im mittleren Donauraum diskutiert, wobei neben der wohl bekannten Bernsteinstraße auch zusätzliche potentielle Routen ins Blickfeld gerückt werden. Neben diesen wissenschaftlichen Ergebnissen aus der Tagung beim Oberleiserberg sei auch die Vernetzung der Latèneexperten/-expertinnen verschiedener zentraleuropäischer und nordadriatischer Regionen hervorgehoben, die sich nicht nur in diesem Band, sondern auch in späteren Folgeveranstaltungen zeigt.

Für die Finanzierung des vorliegenden international begutachteten Bandes danke ich dem Fond zur Förderung der wissenschaftlichen Forschung (FWF) sowie dem Land Niederösterreich (Gruppe Kultur, Wissenschaft und Unterricht). Mein weiterer Dank gilt Estella Weiss-Krejci für die Koordination der Publikationsarbeiten sowie ihr und Ulrike Schuh für die gründliche Redaktion. Schließlich ist den beiden Herausgebern nicht nur besonders $\mathrm{zu}$ danken, sondern es seien ihnen auch interessierte Leser $\mathrm{zu}$ wünschen, die die hier angestoßenen Diskussionen aufgreifen und fortsetzen mögen.

Barbara Horejs

Direktorin des Instituts für Orientalische und Europäische Archäologie

Wien, Februar 2016 
